\title{
A Business Process Modeling Notation Extension for Risk Handling
}

\author{
Bartosz Marcinkowski and Michal Kuciapski \\ University of Gdansk, Department of Business Informatics \\ Piaskowa 9, 81-864 Sopot, Poland \\ \{bartosz.marcinkowski, michal.kuciapski\}@ug.edu.pl
}

\begin{abstract}
During the years of prosperity, numerous organizations neglected numerous aspects of risk management. As systematic approach to handling identified risks is crucial to achieving success by the organization, modern business modeling standards and techniques are supposed to take risk-related features into account. The article is devoted to elaborating and exemplifying an extension aimed at risk handling for OMG's Business Process Modeling Notation (BPMN), one of the most prospective standards for business process modeling. After an introduction, key risk management concepts are discussed. Section 3 discusses extensions introduced within BPMN meta-model, while section 4 exemplifies proposed concepts. The article is concluded with a summary.
\end{abstract}

Keywords: Business Process Modeling Notation, Risk Management, BPMN Extension.

\section{$1 \quad$ Introduction}

Continuous and lasting for decades evolution of all-purpose and domain-centric notations resulted in creating modern modeling standards, such as Unified Modeling Language (UML), Business Process Modeling Notation (BPMN) or BPMS method, integrated in ADONIS business management solution. There is however plenty of room for further improvement. Needless to say, a few BPMN extensions were proposed by academic community and business modeling community.

Early versions of BPMN - although often classified by practitioners as powerful on their own (comp. Harrison-Broninski, 2006) - were enriched with business process goals as well as performance measures by Korherr and List (2007). (Rodriguez, Fernandez-Medina and Piattini, 2007) presented a BPMN 1.0 meta-model with core element and extension that allowed incorporating security requirements into Business Process Diagrams aimed at increasing the scope of the expressive ability of business analysts. (Magnani and Montesi, 2009) addressed relevant limitations of BPMN regarding weak data representation capabilities in comparison to competing standards, designed or adapted to business process modeling needs - such as ADONIS BPMS method or OMG's Unified Modeling Language profiles and custom approaches (comp. Przybylek, 2006). Some of the concepts proposed were included later in 
BPMN 2.0 specification (Object Management Group BPMN, 2011), which was a significant revision that led the emerging standard the UML-alike multi-diagram way (comp. Wrycza, Marcinkowski and Wyrzykowski, 2005). (Stroppi, Chiotti and Villarreal, 2011) offered solutions to strengthen the resource perspective of a business process model elaborated using BPMN 2.0 in order to improve the communication of resource structure, authorization and work structure between business analysts and technical developers. (Zor, Schumm and Leymann, 2011) enhance BPMN 2.0 modeling capabilities within manufacturing domain.

Business Process Modeling Notation extension capabilities are to be used by Object Management Group itself as a UML Profile for BPMN Processes Request For Proposal document (Object Management Group RFP, 2011) was issued. Having that said, the profile is not intended to develop notational capabilities but to provide a mapping between BPMN semantics and the profiled UML semantics as well as define XSLT transforms between the UML XMI for the profile and the BPMN 2 XSD and QVT transforms between the UML and BPMN 2 meta-models.

During the years of prosperity, numerous organizations neglected numerous aspects of risk management. As systematic approach to handling identified risks is crucial to achieving success by the organization, modern business modeling standards and techniques are supposed to take risk-related features into account. The article is devoted to elaborating an extension aimed at risk handling for Business Process Modeling Notation, one of the most prospective standards for process modeling.

\section{Basic Concepts of Risk Management}

According to (ISACA, 2006), risk management is the process of identifying vulnerabilities and threats to the information resources used by an organization in achieving business objectives, and deciding what countermeasures, if any, to take in reducing risk to an acceptable level, based on the value of the information resource to the organization. Risk itself is defined as the combination of the probability of an event and its consequence (International Organization for Standardization, 2009).

Risks may be divided into (International Business Machines Corporation, 2007):

- business-driven risks, strategic in nature and aimed at protecting the business and keeping it accessible whenever and from whoever in support of continuous business operations as well as compliance with industry and government regulations;

- data-driven risks, dealing with the availability of data and information in all of its different forms as used by the organization, including paper-based data;

- event-driven risks, focusing on actual events that create risk to business continuity and viability, such as natural disasters, thefts and IT attacks.

It is risk mitigation procedure that is one of the relevant aspects of risk management to be included in the elaborated extension. Based on the canon of literature in the field of risk management, four standard ways to handle risk are commonly acknowledged (comp. DeLoach and Temple, 2000): 
- reduce,

- retain,

- avoid,

- transfer.

(Husdal, 2009) proposes to set up a wider framework of risk management by discussing exploit and ignore strategies and adding it to the list.

\section{Risk Handling in BPMN - Standard Features and Extensions}

Distinctive features of BPMN include, in particular, very extensive semantics of events. In addition to the division of events into start, intermediate and end ones, the notation development team proposed twelve types of events, distinguished events that are thrown and caught as well as proposed the possibility of interrupting or continuing the flow of source activity at the time of event occurrence. It is the error event that is particularly important type of event from the perspective of risk management. Such event type points to an exception in the underlying activity. The functionality of the described event may in fact be used to assign identified risks to processes, subprocesses or activities to which these risks apply. Indication of potential points of error and how to design their handling is therefore the starting point of expansion of the standard BPMN business process diagram.

BPMN standard, however, does not support formal specification of the identified risks. A business analyst can attempt fulfilling that task by introducing text annotations, which are among the build-in BPMN artifacts, but it is a technique of low clarity and precision. Therefore, the standard was extended with the modeling category of risk factor, characterizing a potential risk in terms of the type, likelihood and impact on business process as a whole. Analogously as in the related publications (Kuciapski and Marcinkowski, 2011), (Kuciapski, 2010) to both the likelihood and impact ranks from 1 to 5 range were assigned, with a value of 1 indicates a low occurrence probability (impact), while the value of 5 - very high occurrence probability (impact).

Risk factor is designed as an independent modeling category due to its complex nature. From the perspective of BPMN meta-model, it was reasonable to assign it to a group of artifacts. Therefore, the RiskFactor is implemented as a child element of Artifact, and supplemented with the additional properties - occurenceProbability and impact (see Fig. 1). A single RiskFactor can be associated with multiple types of risks, but the specification of at least one RiskType is mandatory. Types of risk are classified as integral parts of risk factors, as highlighted with the use of the composition. RiskType is treated in terms of an abstract modeling category. Proposed extension of the standard recognizes five types of risks, but this list may be expanded according to the needs of the end user. Each proposed type of risk is assigned an individual notation. PhysicalResourceRisks can be related to a list of Resources, as HumanResourceRisk - a list of Participants.

Risk factors are assigned to BPMN sequence flows. For this purpose, a standard modeling category of association or placement directly by the relevant sequence flow 
is used. It should be emphasized that with version 2.0 of the BPMN notation, an Association is distinguished from a DataAssociation, hence the association in the current version of the standard is not document-oriented. A single identifiable RiskFactor can be attributed to multiple SequenceFlows. Naturally, from the standpoint of a sequence flow, binding risk factors is optional. The extension also includes the possibility of decomposing risk factors onto component risk factors.

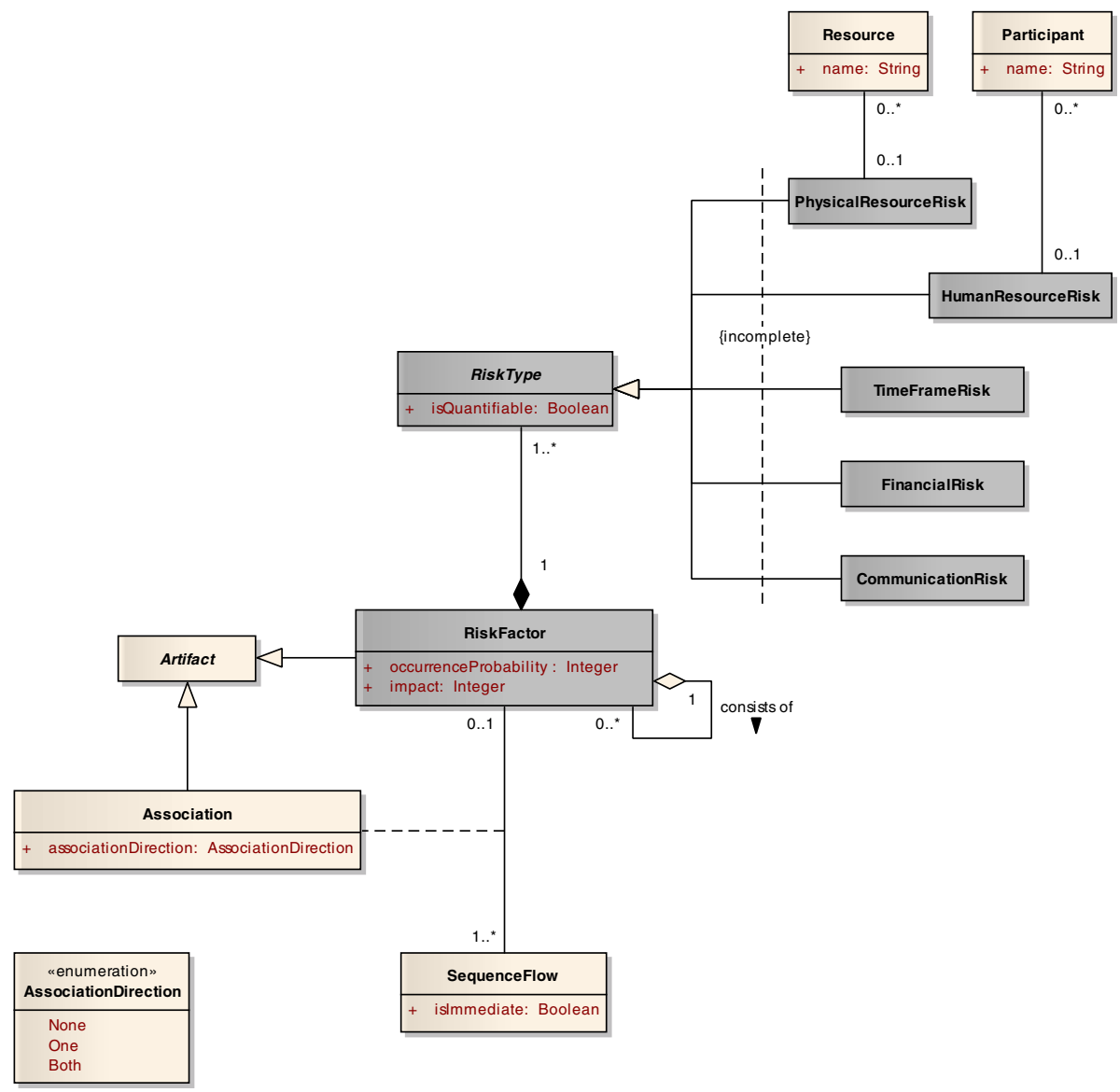

Fig. 1. Risk factors and BPMN meta-model

Each identified risk ought to be assigned with a solution. In the proposed extension, tasks dedicated to handle risk factors are distinguished from tasks forming a natural flow of process or sub-process. In order to achieve that, BPMN meta-model is supplemented with additional kind of Task, i.e. RiskHandler, along with accompanying markup notation (Fig. 2). In order to provide basic compatibility with ADONIS risk analysis process extension for proprietary BPMS notation, both 
RiskHandlers and RiskFactors introduce icons elaborated within mentioned solution. Since a Task is a special case of an Activity, RiskHandler inherits the characteristics of activities. In the context of risk management the significance of Resources assigned to Activities - Contractors in particular - should be emphasized.

It is the mitigationMethod that is an integral property of a RiskHandler. Based on the list of strategies included in section 2 of the current article, six ways to handle risk are proposed: Reduce, Retain, Avoid, Transfer, Exploit and Ignore. As the method is expressed as a String, an enumeration called RiskMitigationMethod is designed for the purpose of storing potential values of the property.

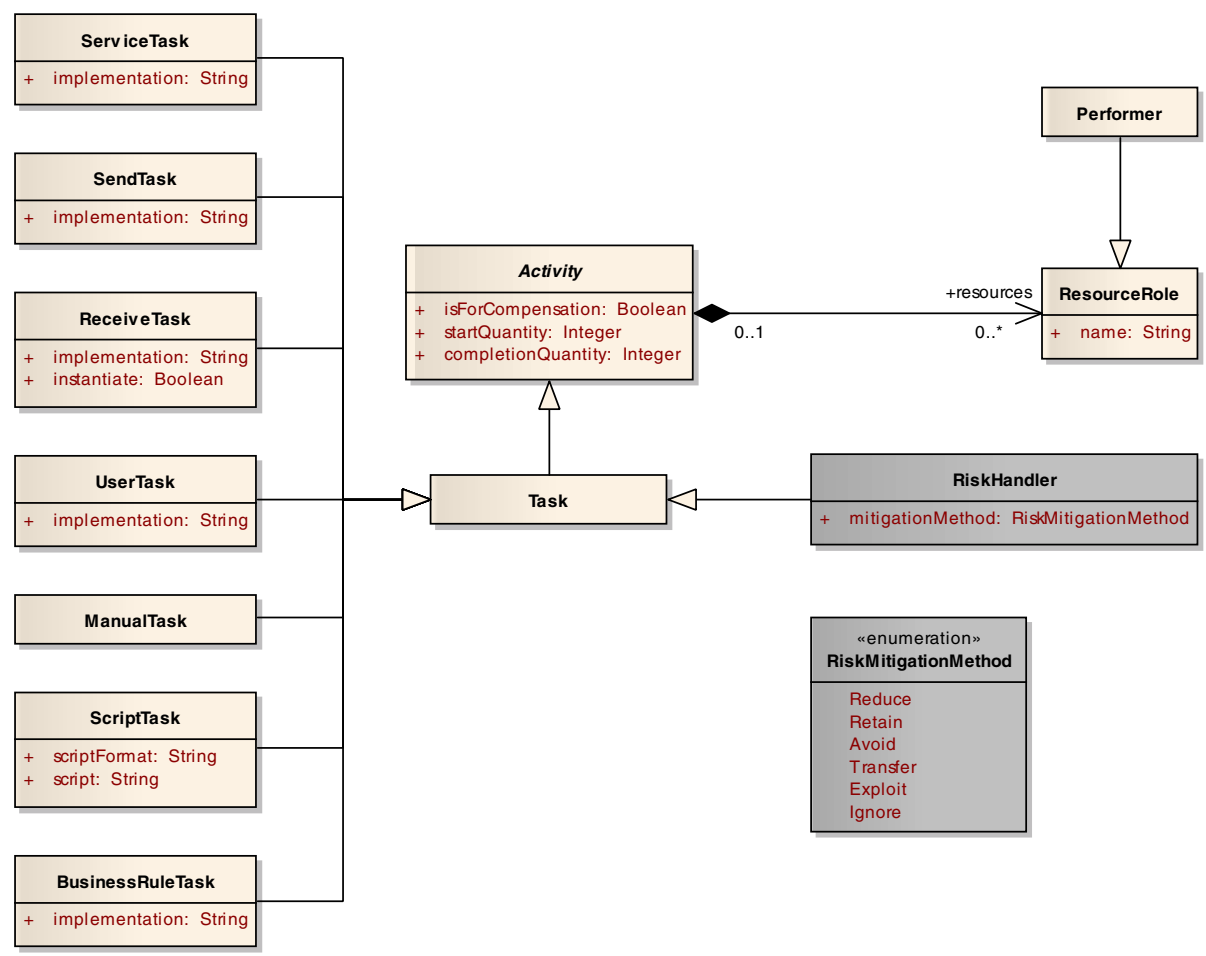

Fig. 2. Risk handler as a child element of BPMN Task

\section{BPMN Extension for Risk Handling Exemplified}

The proposed extension was tested within business process modeling project, involving specification of diverse business processes for real estate developer companies. It is a sub-process that illustrates the procedure for managing architectural contests that is the business functionality selected for the current paper (Fig. 3). 


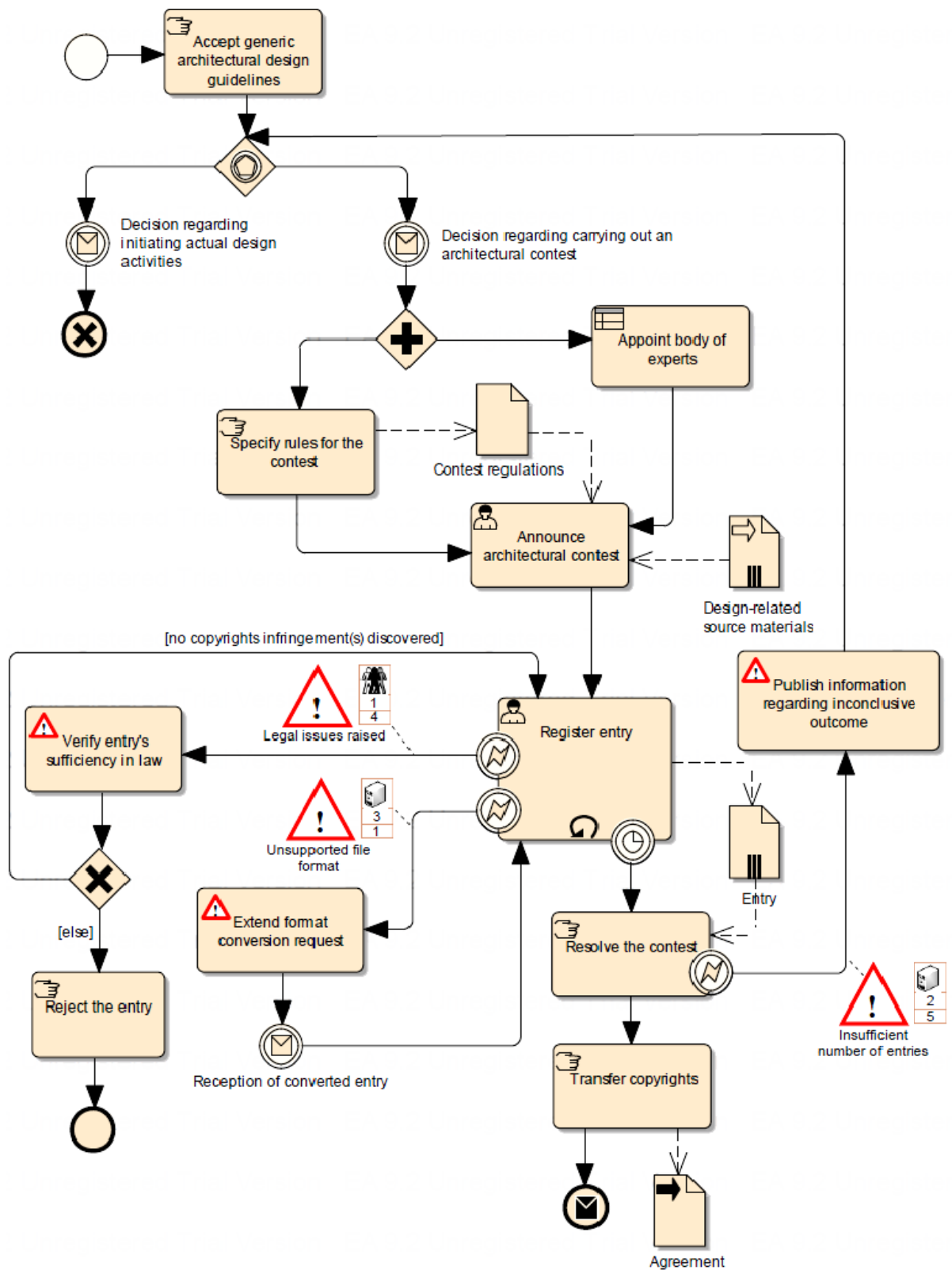

Fig. 3. Procedure for managing architectural contests developed using BPMN Extension for Risk Handling

Analysis of the initial BPMN diagram reveals that it is legitimate to consider a number of risks that the company, responsible for the investment, should foresee in the context of conducting the architectural contest. Thus, risks itemized by the 
company included the risk of insufficient number of entries to the contest, receiving entries that are produced using non-standard toolkits (and thus containing files in nonsupported graphics formats), or occurrence of legal uncertainties as to the authorship of the project. These risks are addressed by risk factors, respectively, Insufficient number of entries, Unsupported file format and Legal issues raised. It should be noted that within the proposed solution of the risk management issues, each identified risk factor is to be attributed to a separate event. Thus, on the border of an activity a lot of intermediate events, responsible for initiating various factors, may be placed.

Two former risk factors are classified as PhysicalResourceRisks, while the latter is classified as a HumanResourceRisk. Risk factor Insufficient number of entries is rather unlikely to occur (rank 2), but has a great impact on the owning sub-process (5). It is Publish information regarding inconclusive outcome that is the task devoted to handling the risk factor. On the other hand, receiving Unsupported file format is more common (rank 3) while having virtually no impact on the sub-process at all (rank 1). Should the risk factor occur, risk handler Extend format conversion request is invoked. Risk factor Raising legal issues is very rare (rank 1) but has significant severity (rank 4). A task Verify entry's sufficiency in law was designed to handle the risk.

\section{Summary}

It was elaborating an extension aimed at risk handling for Business Process Modeling Notation that was the goal of the current article. As BPMN functionality for risk modeling is very limited, even basic risk-oriented framework required introducing custom modeling categories, i.e. RiskFactors, RiskTypes, RiskHandlers as well as RiskMitigationMethods. Owing to the consistent practice of publishing meta-models for standards maintained by Object Management Group, the categories were seamlessly integrated with BPMN meta-model and designed so that the subsequent expansion was possible. Practical applications of proposed extension were exemplified by illustrating the procedure for managing architectural contests.

\section{References}

1. DeLoach, J.W., Temple, N.: Enterprise-wide Risk Management: Strategies for Linking Risk and Opportunity. Financial Times Prentice Hall (2000)

2. Harrison-Broninski, K.: The Future of BPM. Part 2 (2006), http: //www.bptrends.com/publicationfiles/ 09-06-ART-FutureBPM2 0 6-Harrison-Broninski.pdf

3. Husdal, J.: The Six Ways of Dealing with Risk (2009), http://www.husdal.com/ 2009/06/13/the-six-ways-of-dealing-with-risk/

4. International Business Machines Corporation: Risk Mitigation for Business Resilience White Paper. A Comprehensive, Best-Practices Approach to Business Resilience and Risk Migration (2007), http://www-935.ibm.com/services/pl/gts/html/ pdf / gmw14000-usen-00.pdf 
5. International Organization for Standardization: ISO Guide 73:2009 (2009), http://www.iso.org/iso/iso_catalogue/catalogue_ics/ catalogue_detail_ics.htm?csnumber $=44651$

6. ISACA: CISA Review Manual 2006. Information Systems Audit and Control Association (2006)

7. Korherr, B., List, B.: Extending the EPC and the BPMN with Business Process Goals and Performance Measures. In: 9th International Conference on Enterprise Information Systems. ACM Press (2007)

8. Kuciapski, M., Marcinkowski, B.: Risk-oriented Modeling in Business Process Specification (in Polish). In: The Risk of Business Ventures. Foundation for University of Gdansk Development (2011)

9. Kuciapski, M.: Risk Management in e-Learning Projects of Courses Development and Implementation. In: Project Management. Selected Issues, Studies and Materials of Polish Society of Knowledge Management (2010)

10. Magnani, M., Montesi, D.: BPDMN: A Conservative Extension of BPMN with Enhanced Data Representation Capabilities. In: Proceedings of CoRR (2009)

11. Object Management Group: Business Process Model and Notation (BPMN) (2011), http: / / www . omg . org/spec / BPMN/

12. Object Management Group: UML Profile for BPMN Processes RFP (2011), http: / /www.omg.org/cgi-bin/doc?ab/10-06-01

13. Przybylek, A.: The Integration of Functional Decomposition with UML Notation in Business Process Modeling. In: 15th International Conference on Information Systems Development (2006)

14. Rodriguez, A., Fernandez-Medina, E., Piattini, M.: A BPMN Extension for the Modeling of Security Requirements in Business Processes. IEICE Trans. Inf. \& Syst. E90-D (2007)

15. Stroppi, L.J.R., Chiotti, O., Villarreal, P.D.: A BPMN 2.0 Extension to Define the Resource Perspective of Business Process Models. In: CIBSE 2011 - Congresso IberoAmericano em Engenharia de Software (2011)

16. Wrycza, S., Marcinkowski, B., Wyrzykowski, K.: UML 2.0 in Information Systems Modeling (in Polish). Helion (2005)

17. Zor, S., Schumm, D., Leymann, F.: A Proposal of BPMN Extensions for the Manufacturing Domain. In: Proceedings of 44th CIRP International Conference on Manufacturing Systems (2011) 